Beitrag zur Kenntniss gewisser, der Sonne vorüberfliegender Körper. Von Herrn Prof. C. H. F. Peters.

In 1727 der Astr. Nachr. wird durch Herrn Abbé Falb cin Brief wieder ans Licht gezogen, den ich vor Jahren an Herrn Professor Erman schrieb über gewisse Körperchen, welche von den Neapolitanischen Astronomen im Hai 1845 die Sonnenscheibe vorüber passirend wahrgenommen worden waren. Erman hatte kurz zuvor damals die Hypothese aufgestellt, dass der Novemberschwarm der Sternschnuppen in seinem entgegengesetzten Knoten zwischen Sonne und Erde durchgehend den von alten Zeiten her berüchtigten Kälterïckfall im Mai, an den Tagen der Heiligen Mamertius, Pancratius und Servatius (11.-13. Mai), verursachen möchten. Ich glanbte daher, dass meinem verehrten Lehrer und Freunde die Mittheilung jener Wabrnehmungen jedenfalls wenigstens interessant sein würde. Eine nähere Aufklärung über dieselben erscheint jetzt aher nöthig, zumal in einer späteren Nummer dieses Journals ( $\mathcal{W}_{\mathbf{0}}$ 1740) Herr Falb, aus Missverständniss, mich als einen der Gewährsmäuner für die kosmische Natur jener Körper anführt, und zu weiterer Auskunft auffordert, - dem hiermit Genüge geschieht.

Ende September desselben Jahres kam ich wieder nach Neapel, und nahm mir vor, die Erscheinung, wenn sie sich wicder darböte, selhst zu untersuchen. Das Folgende enthält, möglichst in Original, was in meinen Tagebüchern darüber sich vorfindet.

1845 Oct.6. Der Custode GiovanniCortese benachrichtigt uns, dass wieder Körperchen vor der Snnne sich zeigen. Es wird notirt: ,cronometro Breguet, passaggio di corpuscoli davanti il sole, sul cartoncino; in presenza dei professori Belli di Pavia, Tugnoli di Mantova, Don Leopoldo del Re e Peters," - :

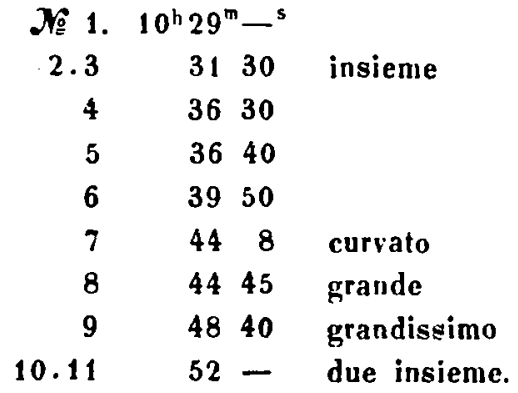

Nachmiltags beobachtete ich allein, - (die Skizzen, welche Richtung und Sehne auf dem Sonnenbilde angeben, lasse ich hier fort):

$\begin{array}{rccl}\text { 16 } 12 . & 2^{\text {h }} 6^{\text {m }} & 1^{\text {s }} & \text { serpeggiante grande; mezzo secondo } \\ 13 & 16:: & \text { grande, } \\ 14 & 3150 & \text { piccolo, dubbio } \\ 15.16 & 428 & 41 & \text { due, uno dopo l'altro, mediocri }\end{array}$

T2 $17.18 .44^{\mathrm{h}} 32^{\mathrm{m}} 50^{\mathrm{s}}$ uno dopo l'altro, mediocri

$19-214023$ tre nello stesso momento, stessa direxione orizzontale

$\begin{array}{rlll}22 & 43 & 14 & \text { unico } \\ 23-28 & 45 & 41 & \text { nolti }(5 \circ 6) \text { nella stessa direzione } \\ 29.30 & 50 & 44 & \text { due, uno dopo l'altro }\end{array}$

3155153 questo ebbe solamente circa la metà della velocità di tutti gli altri, ch'erano in questo rispetto presso a poco uguali.

325448 lento, curvato, in senso opposto

335644 nero, orizzontale, velocità come il più gran numero

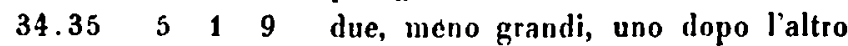
$\begin{array}{llll}36 & 1 & 28 & \text { più grande. }\end{array}$

Nota. A $5^{\mathrm{h}} 9^{\mathrm{m}}$ il oole tramonta dictro i Camaldoli. - Tutti i corpuscoli dopo mezzodi ebbero la direzione pressochè orizzontale. Molte coppie! perche? evvi istinto sociale? -

Oct. 12. Cronometro Breguet: corpuscoli sul cartoncino come prima. Le nuvole muovono dall'Ovest, un poco dal Nord.

J2 $37.8^{11} 39^{m} 45^{s}$ direnione NE, grande, mal distinto

$3844^{4} \mathrm{SO}$, durata $1{ }^{\prime} 5$, ben determinato

394255 SSO, grande, mal determinato

404641 ENE, lucente fuori dello spettro

414750 OSO, $1^{\text {s }}$ sullo spettro

$42559 \mathrm{~S}$, veloce, grande Interruzione da nuvole.

0ct. 17. Nach Beohachtungen von Sonnenflecken (angestellt von $1^{\text {h }} 5^{\text {th }}$ bis $1^{\text {h }} 30^{\text {th }}$ ) folgende Anmerkung: Mentre ch'io determinava queste macchic all' equatoriale, passavano molti corpuscoli, chi più chi meno veloci. Di alcuni poteva bene accorgermi coned'uno spiegarsi di ale, - non v'è dubbio cherano uccelli. Alcuni vedonsi col vetro colorito ancora fuori del disco solare. L'oculare era aggiustato sul sole; il cielo era purissimo, e le macchie si mostravano nella massima hellezza.

Oct. 18. Tra le ore 9-10 della sera. Col cannocchiale aggiustato sulla luna, ho contato in un quarto d'ora 9 corpuscoli. Il settimo era grande, andava orizzontalmente, di sotto e di sopra allargandosi e stringendosi. L'otfavo ed il nono apparvero distinfamente: - erano uccelli, dei quali distinguevasi tutto l'abito: coda, testa e le ale. Tutti passavano, davanti il disco lunare, da tramontana a mezzogiono, più o meno parallelmente all'orizzonte. Uno ne viddi ancora fuori del disco, come un oggetto nero. - La notte è serenissima, ed il vento tutto calmato. - 
Nach diesen Erfahrungen fühle ich mich nicht reranlasst, die Beobachtungen üher diese Gegenstände weiter fortzusetzen. Es ist meine Ueberzeugung, dass, was im Mai vorher gesehen worden, ganz derselben! Natur war, trotz der Versicherungen von ,scharfer Begräinzung" u. s. w. Von den Zugvögeln ist es besonders die Wachtel (quaglia), welche im Frühjahr und im Ilerbst in ungebeuren Scharen das Neapolitanische Gebiet durchzieht. Gerade im Mai ist der Fang derselben in den Netzen, welche auf dem Bergrücken oberbalb Sorrento zwischen boheo Stangen vertical ausgespannt sind, so ergiehig.

Auch mit dem, was Messier gesehen (Arago Astr. Pop. ed. Barral IV., pag. 321: „Messier rapporte que le 17 Juin 1777, vers midi, il vit passer sur le Soleil, pendant cing minutes, un nombre prodigieux de globules noirs" - ) dürfte es wohl eine ganz äbnlicbe Bewandtniss baben, und nichts Kosmisches darin zu suchen sein. -

Hamilton College, März 1869. C. H. F. Peters.

\section{Beobachtungen des Planeten (108) Hecuba auf der Bilk-Düsseldorfer Sternwarte.}

1869 April 4, $9^{1 i} 53^{m} 41^{\prime} 9$ mittl. Zt. Bilk-D. AR 团 $=12^{\mathrm{k}} 4^{\mathrm{m}} 38^{\circ} 52$, Decl. $108=-2^{\circ} 17^{\prime} 8^{\prime \prime} 7 \quad 12$ Vergl. mit $* b(9)$

$1894939,5=:=\quad=12031,12=-15813,1 \quad 12=-13=e(7)$

Die Vergleichsterne purden 80 angenommen:

\begin{tabular}{|c|c|c|c|}
\hline & & Grüsse. & Scheinb. Ort für den Benb. Tag. \\
\hline April & $\boldsymbol{b}$ & 9 & $182^{\circ} 21^{\prime} 32^{\prime \prime} 1$ \\
\hline & $c$ & 7 & $-15810,5$ \\
\hline
\end{tabular}

Den Namen "Hecuba" hat einer der Curatnren der hiesigen Sternwarte, Herr Dr. Franz Meinen, Director der Realschule in Düsseldorf gütigst ausgewählt.

Bilk - D üsseldorf, 1869 April 19.

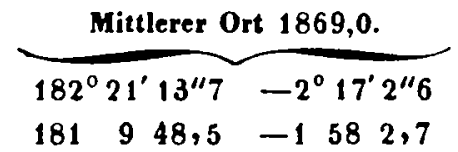

Bei der Proserpina-Beobachtung 1868 Nov. 19 in J6 1752 der Astr. Nachr. sind die Secunden der Rectascension zu lesen $10^{\prime} 35$ statt $19^{9} 35$.

R. Luther.

\section{Meridian-Beobachtungen des Planeten (108) an der Sternwarte in Leiden.}

Nachdem Herr Dr. N. M. Kam den neu entdeckten Planeten am Refractor aufgefunden batte, ist derselbe von Herrn Dr. C. L. F. Kampf, an zwei Abenden, wie folgt, am Meridiankreise beobachtet:

1869 April 11, $10^{\mathrm{h}} 39^{\mathrm{m}} 11^{\mathrm{s}}$ mittl. Zt. Leidea. $\alpha=4^{\mathrm{h}} 59^{\mathrm{m}} 50^{\mathrm{s}} 26, \delta=-1^{\circ} 55^{\prime} 11^{\prime \prime} 78$

$1310305=:=\quad=\quad=5836,19=2323,48$

Herr Dr. Kampf bemerkt, dass dic ungünstige Luft und die Schwäche des Planeten keine sichere Bestimmung zugelassen haben, dass aber doch ohne $Z$ weifel das an beiden Tagen beobacbtete Object der Planet gewesen ist.

Lei de n, 1869 April 19.

F. Kaiser.

\section{Berich t i gu n gen.}

T6 1744, pag. 246 , Zeile 13 von unten, lies $12^{\mathrm{h}}$ statt $12^{\mathrm{m}}$.

$=1744,=247,=15=$ oben, lies: erhellt aus den zablreichen Angaben.

$=1745,=262$ hei $U S$ agittarii und pag. 263 bei $X$ Sagittarii stebt 2 Mal October 1866 anstatt Ort für 1866.

$=1745,=271$, Zeile 2 von oben, lies 1868 statt 1867.

I $\mathrm{n} \mathrm{h}$ a I t .

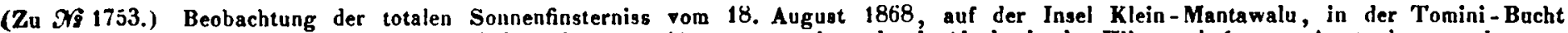
(Ostküste Celebes), von Herrn Dr. J. A. C. Oudemuns. (Auszog auq einer der $k$. Akademie der Wissenschaften zu Amsterdam angebotenen Abhandlung.) 1. - Beobachtungen der Planeten (101) und (106) auf der Sternwarte zu Lund. 7. - Equatorial Observations made at the Durham Observatory, June to December 1868. 11. - Schreiben des Herrn Dr. Wrinnecke an den Herausgeber. 13. - Beobachtungen der Planeten (108) und dos Winnecke'schen Cometen auf der Leipziger Sternwarte. 15. - Beobachtungen des Winnecke'schen Cometen auf der Bonner Sternwarte. 15. - 